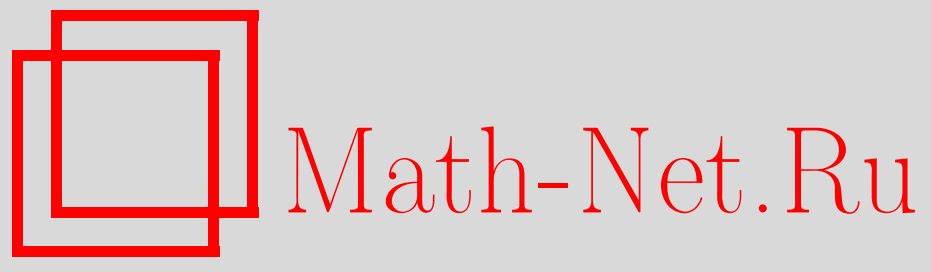

Т. С. Мардвилко, А. А. Пекарский, Прямая и обратная теоремы рациональной аппроксимации в пространстве Бергмана, Матем. сб., 2011, том 202, номер 9, 77-96

DOI: https://doi.org/10.4213/sm7742

Использование Общероссийского математического портала Math-Net.Ru подразумевает, что вы прочитали и согласны с пользовательским соглашением http: //www. mathnet.ru/rus/agreement

Параметры загрузки:

IP: 34.227 .88 .159

26 апреля 2023 г., 14:20:19

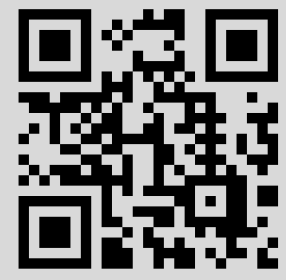




\author{
Т. С. Мардвилко, А. А. Пекарский
}

\title{
Прямая и обратная теоремы рациональной аппроксимации в пространстве Бергмана
}

Для положительных чисел $p$ и $\mu$ через $A_{p, \mu}$ обозначим пространство Бергмана аналитических в полуплоскости $\Pi:=\{z \in \mathbb{C}: \operatorname{Im} z>0\}$ функций. Для $f \in A_{p, \mu}$ введем $R_{n}(f)_{p, \mu}$ - наилучшее приближение рациональными функциями степени не выше $n$. Пусть, кроме того, $\alpha \in \mathbb{R}$ и $\tau>0$ таковы, что $\alpha+\mu=\frac{1}{\tau}-\frac{1}{p}>0$ и $\frac{1}{p}+\mu \notin \mathbb{N}$. Тогда согласно основному результату работы множество функций $f \in A_{p, \mu}$, удовлетворяющих условию

$$
\sum_{n=1}^{\infty} \frac{1}{n}\left(n^{\alpha+\mu} R_{n}(f)_{p, \mu}\right)^{\tau}<\infty,
$$

совпадает с пространством Бесова $B_{\tau}^{\alpha}$ аналитических в П функций.

Библиография: 23 названия.

Ключевые слова: прямые и обратные теоремы рациональной аппроксимации, неравенства типа Бернштейна, неравенства типа Джексона, пространства Бергмана, пространства Бесова.

\section{§ 1. Введение. Основные результаты}

Через $L_{p}(\mathbb{R})$ обозначим пространство комплексных измеримых в смысле Лебега функций $f$ на $\mathbb{R}$ с конечной квазинормой

$$
\|f\|_{L_{p}(\mathbb{R})}=\left(\int_{\mathbb{R}}|f(x)|^{p} d x\right)^{1 / p}, \quad 0<p<\infty .
$$

Введем также $L_{p, \mu}(\Pi)$ - пространство комплексных измеримых относительно плоской меры Лебега $m_{2}$ в полуплоскости

$$
\Pi:=\{z \in \mathbb{C}: \operatorname{Im} z>0\}
$$

функций $f$, для которых конечна квазинорма

$$
\|f\|_{L_{p, \mu}(\Pi)}=\left(\int_{\Pi}(\operatorname{Im} z)^{p \mu-1}|f(z)|^{p} d m_{2}(z)\right)^{1 / p}, \quad p>0, \quad \mu>0 .
$$

Подпространство $L_{p, \mu}(\Pi)$, состоящее из аналитических в П функций, - пространство Бергмана, обозначим через $A_{p, \mu}:=A_{p, \mu}(\Pi)$. При этом для $f \in A_{p, \mu}$ полагаем $\|f\|_{A_{p, \mu}}=\|f\|_{L_{p, \mu}(\Pi)}$.

Для $0<\tau<\infty$ и $\alpha \in \mathbb{R}$ введем пространство Бесова $B_{\tau}^{\alpha}$ аналитических в П функций $f$, удовлетворяющих условию

$$
\|f\|_{B_{\tau}^{\alpha}}=\left\|f^{(s)}\right\|_{A_{\tau, s-\alpha}}<\infty, \quad s=[\alpha]+1,
$$


где $[\alpha]$ - целая часть $\alpha$. Здесь, как обычно, $f^{(0)}=f, f^{(s)}$ при $s \geqslant 1$ есть $s$-я производная $f$, а $f^{(s)}$ при $s<0$ есть $(-s)$-я первообразная функции $f$, однозначно определяемая условием $f^{(s)}(z) \rightarrow 0$ при $\operatorname{Im} z \rightarrow+\infty$. Функционал $\|\cdot\|_{B_{\tau}^{\alpha}}$ на $B_{\tau}^{\alpha}$ задает квазинорму при $[\alpha]+1 \leqslant 0$, т.е. при $\alpha<0$. Если же $[\alpha]+1 \geqslant 1$, т.е. $\alpha \geqslant 0$, то функционал $\|\cdot\|_{B_{\tau}^{\alpha}}$ на $B_{\tau}^{\alpha}$ является полуквазинормой, так как $\|f\|_{B_{\tau}^{\alpha}}=0$ тогда и только тогда, когда $f$ является алгебраическим многочленом степени не выше $[\alpha]$. В $\S 2$ будет показано, что при $\alpha \in\left[0, \frac{1}{\tau}\right)$ функционал $\|\cdot\|_{B_{\tau}^{\alpha}}$ также будет квазинормой, если ограничиться функциями $f$ такими, что $f(z) \rightarrow 0$ при $\operatorname{Im} z \rightarrow+\infty$. В настоящей статье именно это и предполагается. То есть в определении пространства $B_{\tau}^{\alpha}$ при $\alpha \in\left[0, \frac{1}{\tau}\right)$ считаем $f(z) \rightarrow 0$ при $\operatorname{Im} z \rightarrow+\infty$. Как известно, если $f \in B_{\tau}^{\alpha}$, то $s=[\alpha]+1$ в (1.1) можно заменить на любое $s>\alpha$. Получаемые при этом квазинормы будут эквивалентны.

Для пространств $B_{\tau}^{\alpha}$ имеет место следующее непрерывное некомпактное вложение (см., например, [1], [2]):

$$
B_{\tau_{1}}^{\alpha_{1}} \hookrightarrow B_{\tau_{0}}^{\alpha_{0}} \quad \text { при } \quad \alpha_{1}-\alpha_{0}=\frac{1}{\tau_{1}}-\frac{1}{\tau_{0}}>0 .
$$

В частности,

$$
B_{\tau}^{\alpha} \hookrightarrow A_{p, \mu} \quad \text { при } \quad \alpha+\mu=\frac{1}{\tau}-\frac{1}{p}>0 .
$$

Здесь и в дальнейшем запись $X \hookrightarrow Y$ означает непрерывное вложение пространства $X$ в пространство $Y$.

Обозначим через $\mathscr{P}_{n}, n=0,1,2, \ldots$, множество алгебраических полиномов степени не выше $n ; \mathscr{R}_{n}=\left\{\frac{p}{q}: p, q \in \mathscr{P}_{n}, q \not \equiv 0\right\}$ - множество рациональных функций степени не выше $n$. Для $f \in A_{p, \mu}$ введем наилучшее рациональное приближение

$$
R_{n}(f)_{p, \mu}:=R_{n}(f)_{A_{p, \mu}}=\inf \left\{\|f-r\|_{A_{p, \mu}}: r \in \mathscr{R}_{n} \cap A_{p, \mu}\right\} .
$$

Как известно, множество всех рациональных функций является всюду плотным в $A_{p, \mu}$. Поэтому из (1.3) следует, что для $f \in B_{\tau}^{\alpha}$ выполняется соотношение $R_{n}(f)_{p, \mu} \rightarrow 0$ при $n \rightarrow \infty$. Основной результат настоящей статьи - теорема $1-$ включает в себя прямую и обратную теоремы рациональной аппроксимации B $A_{p, \mu}$.

Теорема 1. Пусть положительные числа $p, \mu, \tau$ и действительное $\alpha$ такови, что $\alpha+\mu=\frac{1}{\tau}-\frac{1}{p}>0 u \frac{1}{p}+\mu \notin \mathbb{N}$. Тогда функиия $f \in A_{p, \mu}$ удовлетворяет условию

$$
\sum_{n=1}^{\infty} \frac{1}{n}\left(n^{\alpha+\mu} R_{n}(f)_{p, \mu}\right)^{\tau}<\infty
$$

в том и толъко том случае, когда $f \in B_{\tau}^{\alpha}$.

Достаточность из теоремы 1 (прямая теорема) имеет место и при $\frac{1}{p}+\mu \in \mathbb{N}$. Доказать достаточность можно методом из работы [3], в которой получены прямые теоремы рациональной аппроксимации в пространствах Харди. Можно также применить атомическое представление (см. [4], [1]) функций из пространства Бергмана. В [4], [1] атомическое представление применяется, в частности, 
для доказательства прямой теоремы рациональной аппроксимации в пространстве Блоха. При доказательстве достаточности из теоремы 1 нами также получено неравенство типа Джексона - теорема 2.

Теорема 2. Пусть $\alpha$-действительное $и p, \mu, \tau$ - положительные числа maкие, что $\alpha+\mu=\frac{1}{\tau}-\frac{1}{p}>0$. Если $f \in B_{\tau}^{\alpha}$, mo

$$
R_{n}(f)_{A_{p, \mu}} \leqslant \frac{c}{n^{\alpha+\mu}}\|f\|_{B_{\tau}^{\alpha}}, \quad n=1,2, \ldots,
$$

где с $>0$ и не зависит от $n u f$.

Необходимость из теоремы 1 (обратная теорема) доказывается методом С.Н. Бернштейна доказательства обратных теорем теории аппроксимации и основана в данном случае на приведенной ниже теореме 4. Кроме того, в случае $1<\tau<\infty$ мы используем метод вещественной интерполяции. Теорема 4 доказывается с использованием теоремы 3 , представляющей также самостоятельный интерес.

ТЕОРема 3. Пусть $р$ и $\mu$ - положительные числа такие, что $\frac{1}{\lambda}=\frac{1}{p}+$ $\mu \notin \mathbb{N}$. Тогда для $r \in \mathscr{R}_{n} \cap L_{p, \mu}(\Pi), n \geqslant 1$, выполняется неравенство

$$
\|r\|_{L_{\lambda}(\mathbb{R})} \leqslant c n^{\mu}\|r\|_{L_{p, \mu}(\Pi)}, \quad c=c(p, \mu)>0 .
$$

Теорема 4. Пусть р и Тогда для $\alpha>-\mu, \frac{1}{\tau}=\alpha+\mu+\frac{1}{p} u r \in \mathscr{R}_{n} \cap A_{p, \mu}, n \geqslant 1$, выполняется неравенство

$$
\|r\|_{B_{\tau}^{\alpha}} \leqslant c n^{\alpha+\mu}\|r\|_{A_{p, \mu}}, \quad c=c(p, \mu, \alpha)>0 .
$$

Ограничение $\frac{1}{p}+\mu \notin \mathbb{N}$ в теоремах 3 и 4 , а также в случае необходимости из теоремы 1 является существенным.

Теоремы 1-4 имеют свои аналоги и для круга. В случае $\mu+\frac{1}{p}<1$ аналоги теорем 1-4 для круга были получены ранее Е. Дынькиным (см. [5]). В частном случае $\mu=\frac{1}{p}$ и $p>2$ также для круга необходимость из теоремы 1 , а также теоремы 3 и 4 были установлены В.Р. Мисюком (см. [6]) одновременно с Е. Дынькиным и независимо от него. Теорема 3 для $\mu=\frac{1}{p}$ и $0<p<1$ была получена недавно первым из авторов (см. [7]). При этом в [7] существенным образом использовались некоторые результаты и приемы из [8]. Этим методы работ [7] и настоящей работы существенным образом отличаются от методов работ [5] и [6]. Доказательства теорем 3 и 4 основаны на дальнейшем развитии методов работ [8], [7].

Основные результаты настоящей статьи анонсированы в [9].

\section{§ 2. Доказательство прямой теоремы и неравенства типа Джексона}

Условимся через $c, c_{1}, c_{2}, \ldots$ обозначать некоторые положительные величины, зависящие лишь от указанных в скобках параметров. Иногда значения параметров будем опускать.

В 55 будут приведены некоторые результаты, касающиеся пространства Бергмана $A_{p, \mu}(G)$ в области $G \neq \mathbb{C}$. В частности, согласно лемме 10 для $f \in$ 
$A_{p, \mu}(\Pi)$ имеет место неравенство

$$
|f(z)| \leqslant \frac{c(p, \mu)}{(\operatorname{Im} z)^{\mu+1 / p}}\|f\|_{A_{p, \mu}}, \quad z \in \Pi .
$$

Из (2.1) вытекает, что требование $f(z) \rightarrow 0$ при $\operatorname{Im} z \rightarrow+\infty$ является корректным для функций $f \in B_{\tau}^{\alpha}(\Pi)$, если $\alpha<\frac{1}{\tau}$.

Для доказательства прямой теоремы и неравенства типа Джексона мы применим полученное Р. Р. Койфманом и Р. Рочбергом (см. [4]) атомическое разложение функций класса $A_{p, \mu}$. Об атомических разложениях и их приложениях также см. работы [1], [2]. Нужным результатам из [4], [1] мы придадим удобную для нас форму и сформулируем в виде теорем 5 и 6. С этой целью введем следующие обозначения.

Для каждого $\theta \in(0,1)$ введем множество $\mathscr{F}_{\theta}$ замкнутых квадратов

$$
Q=\left\{z: \theta^{p+1} \leqslant \operatorname{Im} z \leqslant \theta^{p} ; q \theta^{p}(1-\theta) \leqslant \operatorname{Re} z \leqslant(q+1) \theta^{p}(1-\theta)\right\}, \quad \text { где } p, q \in \mathbb{Z} .
$$

Очевидно, эти квадраты могут пересекаться лишь по границам и объединение их всех есть П. Квадраты семейства $\mathscr{F}_{\theta}$ будем нумеровать в каком-либо порядке с помощью натуральных чисел: $\mathscr{F}_{\theta}=\left\{Q_{k}\right\}_{k=1}^{\infty}$. Через $z_{k}$ обозначим центр квадрата $Q_{k}$.

TeOpema 5. Пусть $\theta \in(0,1), p>0, \mu>0 u$

$$
\varkappa>\max \left\{1, \frac{1}{p}\right\}+\mu \text {. }
$$

Тогда для любой последовательности $\left\{u_{k}\right\}_{k=1}^{\infty}$, удовлетворяющей условию

$$
\sum_{k=1}^{\infty}\left|u_{k}\right|^{p}<\infty
$$

ряд

$$
\sum_{k=1}^{\infty} u_{k} \frac{\left(\operatorname{Im} z_{k}\right)^{\varkappa-(\mu+1 / p)}}{\left(z-\bar{z}_{k}\right)^{\varkappa}}, \quad z \in \Pi
$$

сходится в $A_{p, \mu}$, а также он сходится равномерно и абсолютно на компактных подмножествах П. При этом сумма $f(z)$ ряда (2.2) удовлетворяет условию

$$
\|f\|_{A_{p, \mu}}^{p} \leqslant c_{1} \sum_{k=1}^{\infty}\left|u_{k}\right|^{p}, \quad c_{1}=c_{1}(p, \mu, \varkappa, \theta)>0 .
$$

Теорема 6. Пусть $p, \mu$ и такие же, как в теореме 5. Тогда существует $\theta_{0}=\theta_{0}(p, \mu, \varkappa) \in(0,1)$ такое, что при каждом $\theta \in\left(\theta_{0}, 1\right)$ любая функиия $f \in A_{p, \mu}$ представима с помощью ряда (2.2) с коэффициентами $\left\{u_{k}\right\}_{k=1}^{\infty}$, удовлетворяющими условию

$$
\sum_{k=1}^{\infty}\left|u_{k}\right|^{p} \leqslant c_{2}\|f\|_{A_{p, \mu}}^{p}, \quad c_{2}=c_{2}(p, \mu, \varkappa, \theta)>0 .
$$


Из теорем 5 и 6 следуют, в частности, вложения (1.2) и (1.3). Используя эти теоремы, легко показать, что квазинормы $\|f\|_{A_{p, \mu}}$ и $\left\|f^{(s)}\right\|_{A_{p, \mu+s}, s \in}$ $(-\mu,+\infty) \cap \mathbb{Z}$, эквивалентны для $f \in A_{p, \mu}$. Следовательно, заменив $s=[\alpha]+1$ в (1.1) на любое целое $s>\alpha$, получим эквивалентную квазинорму для пространства $B_{\tau}^{\alpha}$.

Нам также понадобятся следующие леммы 1 и 2. Лемма 1 является фрагментом доказательства теоремы 1.3 .1 из [10]. Лемма 2 следует из неравенства

$$
(x+y)^{\theta} \leqslant x^{\theta}+y^{\theta}, \quad \text { где } x \geqslant 0, y \geqslant 0,0<\theta \leqslant 1 .
$$

Лемма 1. Пусть $0<q<1,\left\{a_{n}\right\}_{n=1}^{\infty}-$ невозрастающая последовательность неотрицательных чисел и $\sum a_{n}^{q}<\infty$. Тогда

$$
\sum_{n=1}^{\infty}\left(\frac{1}{n} \sum_{k=n}^{\infty} a_{k}\right)^{q} \leqslant c(q) \sum_{n=1}^{\infty} a_{n}^{q}
$$

Лемма 2. Пусть $0<\alpha<\beta<\infty,\left\{a_{k}\right\}_{k=1}^{\infty}-$ последовательность неотрицательных чисел и $\sum a_{k}^{\alpha}<\infty$. Тогда

$$
\left(\sum_{k=1}^{\infty} a_{k}^{\beta}\right)^{1 / \beta} \leqslant\left(\sum_{k=1}^{\infty} a_{k}^{\alpha}\right)^{1 / \alpha}
$$

Достаточность из теоремы 1 (прямая теорема), а также теорема 2 являются очевидными следствиями теоремы 7.

ТеОрема 7. Пусть положительные числа $p, \mu, \tau$ и действительное $\alpha$ такови, что

$$
\alpha+\mu=\frac{1}{\tau}-\frac{1}{p}>0 .
$$

Eсли $f \in B_{\tau}^{\alpha}$, mo

$$
\sum_{n=1}^{\infty} \frac{1}{n}\left(n^{\alpha+\mu} R_{n}(f)_{p, \mu}\right)^{\tau} \leqslant c\|f\|_{B_{\tau}^{\alpha}}^{\tau}, \quad c=c(p, \mu, \tau, \alpha)>0 .
$$

ДоказАтельство. Положим $s=\max \{0,[\alpha]+1\}$, где $[\alpha]-$ целая часть числа $\alpha$. Далее, выберем $\varkappa \in \mathbb{N}$, удовлетворяющее условию $\varkappa>1+\mu+s+\frac{1}{p}$. Очевидно, будет выполнено также неравенство $\varkappa>\max \left\{1, \frac{1}{\tau}\right\}+s-\alpha$. Поскольку $f \in B_{\tau}^{\alpha}$, то $f^{(s)} \in A_{\tau, s-\alpha}$. Согласно теореме 6 функцию $f^{(s)}$ при некотором $\theta_{1} \in(0,1)$ можно представить в виде ряда

$$
f^{(s)}(z)=\sum_{k=1}^{\infty} u_{k} \frac{\left(\operatorname{Im} z_{k}\right)^{\varkappa-(s-\alpha)-1 / \tau}}{\left(z-\bar{z}_{k}\right)^{\varkappa}}, \quad z \in \Pi .
$$

Здесь $\left\{z_{k}\right\}_{k=1}^{\infty}-$ соответственно центры квадратов $\left\{Q_{k}\right\}_{k=1}^{\infty}$ семейства $\mathscr{F}_{\theta_{1}}$. При этом коэффициенты $u_{k}$ ряда (2.5) таковы, что

$$
\sum_{k=1}^{\infty}\left|u_{k}\right|^{\tau} \leqslant c_{1}\left\|f^{(s)}\right\|_{A_{\tau, s-\alpha}}^{\tau} \leqslant c_{2}\|f\|_{B_{\tau}^{\alpha}}^{\tau} .
$$

Будем считать, что квадраты $Q_{k}$ пронумерованы таким образом, что последовательность $\left\{\left|u_{k}\right|\right\}_{k=1}^{\infty}$ не возрастает. 
Из (2.1) находим, что

$$
\left|f^{(s)}(z)\right| \leqslant \frac{c_{3}}{(\operatorname{Im} z)^{s-\alpha+1 / \tau}}\|f\|_{B_{\tau}^{\alpha}}, \quad z \in \Pi .
$$

Из этого неравенства и теоремы 5 следует, что ряд (2.5) можно почленно проинтегрировать $s$ раз вдоль вертикальных лучей $[z, z+i \infty), z \in \Pi$. С учетом условия (2.3) получим

$$
f(z)=b \sum_{k=1}^{\infty} u_{k} \frac{\left(\operatorname{Im} z_{k}\right)^{(\varkappa-s)-(\mu+1 / p)}}{\left(z-\bar{z}_{k}\right)^{\varkappa-s}}, \quad z \in \Pi,
$$

где $b=(-1)^{s} \frac{(\varkappa-s-1) !}{(\varkappa-1) !}$.

Поскольку $p>\tau$, то из (2.6) и леммы 2 следует сходимость ряда $\sum\left|u_{k}\right|^{p}$. Тогда согласно теореме 5 ряд (2.7) сходится в $A_{p, \mu}$. Для $n \in \mathbb{N}$ положим $r_{n}(z)=0$ при $n<\varkappa-s$ и

$$
r_{n}(z)=b \sum_{1 \leqslant k \leqslant n /(\varkappa-s)} u_{k} \frac{\left(\operatorname{Im} z_{k}\right)^{(\varkappa-s)-(\mu+1 / p)}}{\left(z-\bar{z}_{k}\right)^{\varkappa-s}} \text { при } n \geqslant \varkappa-s .
$$

Очевидно, $r_{n} \in \mathscr{R}_{n} \cap A_{p, \mu}$ и, следовательно, $R_{n}(f)_{p, \mu} \leqslant\left\|f-r_{n}\right\|_{A_{p, \mu}}$. Для оценки последней квазинормы применим теорему 5 . В результате получим

$$
R_{n}(f)_{p, \mu} \leqslant c_{5}\left(\sum_{k>n /(\varkappa-s)}\left|u_{k}\right|^{p}\right)^{1 / p} .
$$

Далее, положим $a_{k}=\left|u_{[k /(\varkappa-s)]+1}\right|, k=1,2, \ldots$. Последовательность $\left\{a_{k}\right\}_{k=1}^{\infty}$ неотрицательна, не возрастает и согласно (2.6)

$$
\sum_{k=1}^{\infty} a_{k}^{\tau} \leqslant c_{6}\|f\|_{B_{\tau}^{\alpha}}^{\tau}
$$

Из (2.8) получим также, что

$$
R_{n}(f)_{p, \mu} \leqslant c_{7}\left(\sum_{k=n}^{\infty}\left|a_{k}\right|^{p}\right)^{1 / p}
$$

В силу (2.3) имеем $\frac{\tau}{p}<1$. Поэтому, используя лемму 1 и соотношения $(2.9)$ и $(2.10)$, получим $(2.4)$ :

$$
\sum_{n=1}^{\infty} \frac{1}{n}\left(n^{\alpha+\mu} R_{n}(f)_{p, \mu}\right)^{\tau} \leqslant c_{8} \sum_{n=1}^{\infty}\left(\frac{1}{n} \sum_{k=n}^{\infty}\left|a_{k}\right|^{p}\right)^{\tau / p} \leqslant c_{9} \sum_{n=1}^{\infty} a_{n}^{\tau} \leqslant c_{10}\|f\|_{B_{\tau}^{\alpha}}^{\tau} .
$$

Теорема доказана.

\section{§ 3. Сравнение квазинорм рациональной функции относительно линейной и плоской мер}

Здесь мы докажем теорему 3. Нам понадобится пространство Харди $H_{p}=$ $H_{p}(\Pi)$ в полуплоскости П. Согласно определению (см. [11]) аналитическая в полуплоскости П функция $f$ принадлежит $H_{p}, 0<p<\infty$, если

$$
\|f\|_{H_{p}}:=\sup _{y>0}\|f(\cdot+i y)\|_{L_{p}(\mathbb{R})}<\infty .
$$


Как известно, если $f \in H_{p}$, то для почти всех $x \in \mathbb{R}$ существует $\lim f(z)=: f(x)$, когда $z \in \Pi$ стремится к $x$ по некасательным по отношению к $\mathbb{R}$ путям. При этом $\|f\|_{H_{p}}=\|f\|_{L_{p}(\mathbb{R})}$. Отметим также для $f \in H_{p}$ неравенство

$$
|f(z)| \leqslant \frac{c(p)}{(\operatorname{Im} z)^{1 / p}}\|f\|_{H_{p}}, \quad z \in \Pi .
$$

Утверждения типа следующей условной леммы 3 имеются также в работах [8], [7].

ЛЕмма 3. Пусть положительные числа $p, \mu$ и у удовлетворяют условию $\frac{1}{\lambda}=\mu+\frac{1}{p}$. Предположим, что для некоторого $p=p_{1}<1$ неравенство

$$
\|r\|_{H_{\lambda}} \leqslant c_{1}(p, \mu) n^{\mu}\|r\|_{A_{p, \mu}}
$$

выполняется для любой функиии $r \in \mathscr{R}_{n} \cap A_{p, \mu}, n \geqslant 1$, с действительными коэффициентами и с полюсами лишь на $\mathbb{R}$. Тогда для всех $p=k p_{1}, k \in \mathbb{N}, u$ всех $r \in \mathscr{R}_{n} \cap L_{p, \mu}(\Pi), n \geqslant 1$, выполняется неравенство

$$
\|r\|_{L_{\lambda}(\mathbb{R})} \leqslant c_{2}(p, \mu) n^{\mu}\|r\|_{L_{p, \mu}(\Pi)}
$$

c некоторой константой $c_{2}(p, \mu)$ вместо $c_{1}(p, \mu)$.

ДокаЗАтельство. Рассмотрим сначала случай $k=1$. Пусть $r \in \mathscr{R}_{n} \cap$ $L_{p, \mu}(\Pi)$ и не все ее полюсы лежат на $\mathbb{R}$. Через $b_{+}$и $b_{-}$обозначим соответственно произведения Бляшке, полюсами которых с учетом кратности являются полюсы $r$, лежащие в полуплоскостях $\operatorname{Im} z>0$ и $\operatorname{Im} z<0$. Если, например, $r$ не имеет полюсов в $\operatorname{Im} z<0$, то полагаем $b_{-}(z) \equiv 1$. Очевидно, что $\infty$ полюсом $r$ не может быть. Для каждого фиксированного $\zeta \in T, T:=\{\zeta:|\zeta|=1\}$, все полюсы рациональной функции

$$
r(z, \zeta):=r(z)\left(b_{+}(z)-\zeta b_{-}(z)\right)^{-1}
$$

лежат на $\mathbb{R}$ и ее степень равна степени $r$. Несложно убедится (или см. [12; теорема 1.7], [7; лемма 1]), что функция

$$
J(p, z):=\int_{\mathrm{T}}\left|b_{+}(z)-\zeta b_{-}(z)\right|^{-p}|d \zeta|
$$

ограничена в $\mathbb{C}$, т.е. $J(p, z) \leqslant \omega(p)$ при $z \in \mathbb{C}$, где $\omega(p)$ больше нуля и зависит лишь от $p=p_{1} \in(0,1)$. Используя теорему Фубини, находим, что

$$
\begin{aligned}
& \int_{T}\left(\int_{\Pi}(\operatorname{Im} z)^{p \mu-1}|r(z, \zeta)|^{p} d m_{2}(z)\right)|d \zeta| \\
& \quad=\int_{\Pi}(\operatorname{Im} z)^{p \mu-1}|r(z)|^{p} J(p, z) d m_{2}(z) \leqslant \omega(p)\|r\|_{L_{p, \mu}(\Pi)}^{p} .
\end{aligned}
$$

Следовательно, существует $\zeta_{0} \in T$ такое, что функция $r_{0}(z):=r\left(z, \zeta_{0}\right)$ удовлетворяет условию

$$
\left\|r_{0}\right\|_{A_{p, \mu}} \leqslant c_{3}(p)\|r\|_{L_{p, \mu}(\Pi)}, \quad c_{3}(p)=\left(\frac{\omega(p)}{2 \pi}\right)^{1 / p} .
$$


Положим $r_{1}(x)=\operatorname{Re} r_{0}(x)$ и $r_{2}(x)=\operatorname{Im} r_{0}(x), x \in \mathbb{R}$. Тогда для $r_{j}, j=1,2$, выполняется соотношение

$$
\left\|r_{j}\right\|_{A_{p, \mu}} \leqslant c_{3}(p)\|r\|_{L_{p, \mu}(\Pi)} .
$$

Для $r_{j}$ согласно предположению выполняется неравенство (3.2). С учетом (3.2), (3.4) и очевидного неравенства $\left|b_{+}(x)-\zeta_{0} b_{-}(x)\right| \leqslant 2, x \in \mathbb{R}$, находим

$$
\begin{aligned}
\left\|r_{j}\left(b_{+}-\zeta_{0} b_{-}\right)\right\|_{L_{\lambda}(\mathbb{R})} & \leqslant 2\left\|r_{j}\right\|_{L_{\lambda}(\mathbb{R})} \leqslant 2 c_{1}(p, \mu) n^{\mu}\left\|r_{j}\right\|_{A_{p, \mu}} \\
& \leqslant 2 c_{1}(p, \mu) c_{3}(p) n^{\mu}\|r\|_{L_{p, \mu}(\Pi)}, \quad j=1,2 .
\end{aligned}
$$

Поскольку $r=\left(r_{1}+i r_{2}\right)\left(b_{+}-\zeta_{0} b_{-}\right)$и $0<\lambda<1$, то в силу (3.5) неравенство $(3.3)$ для любой функции $r \in \mathscr{R}_{n} \cap L_{p, \mu}(\Pi)$ будет выполняться с константой $c_{2}(p, \mu)=$ $2^{1 / \lambda} c_{1}(p, \mu) c_{3}(p)$ вместо $c_{1}(p, \mu)$.

Покажем сейчас справедливость (3.3) для показателей $p=p_{k}:=k p_{1}, k=$ $2,3, \ldots$. Пусть $r \in \mathscr{R}_{n} \cap L_{p, \mu}(\Pi)$. Тогда $r^{k} \in \mathscr{R}_{n k} \cap L_{p_{1}, k \mu}(\Pi)$ и, следовательно, согласно рассмотренному случаю $k=1$ выполняется неравенство

$$
\left\|r^{k}\right\|_{L_{\sigma_{k}}(\mathbb{R})} \leqslant c_{2}\left(p_{1}, k \mu\right)(n k)^{k \mu}\left\|r^{k}\right\|_{L_{p_{1}, k \mu}(\Pi)}, \quad \frac{1}{\sigma_{k}}:=k \mu+\frac{1}{p_{1}} .
$$

Далее, положим $\frac{1}{\lambda_{k}}=\mu+\frac{1}{k p_{1}}$ и заметим, что

$$
\left\|r^{k}\right\|_{L_{\sigma_{k}}(\mathbb{R})}=\|r\|_{L_{\lambda_{k}}(\mathbb{R})}^{k}, \quad\left\|r^{k}\right\|_{L_{p_{1}, k \mu}(\Pi)}=\|r\|_{L_{p_{k}, \mu}(\Pi)}^{k} .
$$

Таким образом, из (3.6) следует нужное неравенство

$$
\|r\|_{L_{\lambda_{k}}(\mathbb{R})} \leqslant c_{4}\left(p_{k}, \mu\right) n^{\mu}\|r\|_{L_{p_{k}, \mu}(\Pi)}
$$

с константой $c_{4}\left(p_{k}, \mu\right)=k^{\mu} c_{2}\left(p_{1}, k \mu\right)$. Лемма доказана.

Далее мы следуем схеме рассуждений, примененной в [8] и [7]. С этой целью введем дополнительные обозначения. Пусть $n$ и $l$ - натуральные числа такие, что $n \geqslant l+1$. Через $\mathscr{R}_{n}^{l}$ обозначим множество функций $r \in \mathscr{R}_{n}$ с действительными коэффициентами и с полюсами лишь на $\mathbb{R}$, причем кратность каждого полюса не выше $l$ и

$$
r(z)=O\left(z^{-l-1}\right) \quad \text { при } \quad z \rightarrow \infty .
$$

Через $\Pi_{m}^{l}, m \geqslant 2, l \geqslant 1$, обозначим множество финитных кусочно полиномиальных на $\mathbb{R}$ функций $\varphi$ степени не выше $l-1$ с $m$ свободными узлами. А именно, $\varphi \in \Pi_{m}^{l}$, если существует $m$ точек (узлов $\varphi$ ) $-\infty<x_{1}<x_{2}<\cdots<x_{m}<+\infty$ таких, что $\left.\varphi\right|_{\left(x_{k}, x_{k+1}\right)} \in \mathscr{P}_{l-1}, k=1,2, \ldots, m-1$, и $\varphi(x)=0$ при $x \in \mathbb{R} \backslash\left[x_{1}, x_{m}\right]$. Значения $\varphi\left(x_{k}\right), k=1,2, \ldots, m$, выбираются произвольно. Ради удобства считаем $\Pi_{1}^{l}=\{0\}$.

Лемма 4 (см. [8]). Если $r \in \mathscr{R}_{n}^{l}$, то существует действительная функиия $\varphi \in \Pi_{m}^{l}, m \leqslant n$, maкая, что

$$
r(z)=\frac{l !}{\pi} \int_{\mathbb{R}} \frac{\varphi(t) d t}{(t-z)^{l+1}}, \quad z \in \mathbb{C} \backslash \operatorname{supp} \varphi .
$$

При этом узлами ч являются полюсы $r$. 
Лемма 5 (см. [8]). Пусть $1<q<\infty, k \in \mathbb{N}, \lambda=\left(k+\frac{1}{q}\right)^{-1}, \varphi \in \Pi_{m}^{l} u$

$$
f(z)=\frac{1}{\pi} \int_{\mathbb{R}} \frac{\varphi(t) d t}{t-z}, \quad z \in \Pi .
$$

Тогда $f^{(k)} \in H_{\lambda}$ и выполняется неравенство

$$
\left\|f^{(k)}\right\|_{H_{\lambda}} \leqslant c(k, q, l) \cdot m^{k} \cdot\|\varphi\|_{L_{q}(\mathbb{R})} .
$$

Мы будем использовать также пространства Бергмана $A_{p, \mu}(G)$ и В. И. Смирнова $E_{p}(G)$ в ограниченной области $G$, граница которой $\partial G$ является спрямляемой кривой Жордана. Определения этих пространств и некоторые связанные с ними неравенства приводятся в §5. Методом, применяемым при доказательстве теоремы 10, можно получить также следующее известное вложение. Пусть $p, q$ и $\mu$ - положительные числа такие, что

$$
\mu+\frac{1}{p}-\frac{1}{q}=: l \in \mathbb{N}, \quad p<q .
$$

Если $f$ аналитична в $\Pi, f(z) \rightarrow 0$ при $\operatorname{Im} z \rightarrow+\infty$ и $f^{(l)} \in A_{p, \mu}(\Pi)$, то $f \in H_{q}(\Pi)$ и выполняется неравенство

$$
\|f\|_{H_{q}} \leqslant c(p, q, \mu)\left\|f^{(l)}\right\|_{A_{p, \mu}} .
$$

Пусть $l \in \mathbb{N}, \varepsilon>0$, функция $f$ аналитична в П и $f(z)=O\left((\operatorname{Im} z)^{-l-\varepsilon}\right)$ при $\operatorname{Im} z \rightarrow \infty$. В этом случае через $f^{(-l)}$ будем обозначать $l$-ю первообразную функции $f$, однозначно определяемую условием $f^{(-l)}(z) \rightarrow 0$ при $\operatorname{Im} z \rightarrow \infty$. В начале $\S 2$ было показано, что $f^{(-l)}$ определена корректно для $f \in A_{p, \mu}$ при $\mu+\frac{1}{p}>l$. Из (3.1) следует, что $f^{(-l)}$ определена корректно для $f \in H_{p}$ при $\frac{1}{p}>l$.

Лемма 6. Пусть $p, q, \mu$ u $l$ maкuе же, как в (3.7), $u f \in A_{p, \mu}(\Pi)$. Тогда $f^{(-l)} \in H_{q}$ и для любого $k \in \mathbb{N}$ существует $\psi \in \Pi_{k}^{l}$ такая, что

$$
\left\|\operatorname{Im} f^{(-l)}-\psi\right\|_{L_{q}(\mathbb{R})} \leqslant \frac{c}{k^{l-\mu}}\|f\|_{A_{p, \mu}},
$$

где с больше нуля и не зависит от $f u k$.

ДокАЗАтельство. В силу (3.8) при $k=1$ можем положить $\psi \equiv 0$. Далее считаем $k \geqslant 2$ и $f \not \equiv 0$. Из (3.8) следует существование $x_{1}, x_{k} \in \mathbb{R}, x_{1}<x_{k}$, удовлетворяющих условию

$$
\left\|f^{(-l)}\right\|_{L_{q}\left(\mathbb{R} \backslash\left(x_{1}, x_{k}\right)\right)} \leqslant \frac{1}{k^{1 / p}}\|f\|_{A_{p, \mu}} .
$$

Далее, можем определить $x_{2}, x_{3}, \ldots, x_{k-1}, x_{1}<x_{2}<x_{3}<\cdots<x_{k-1}<x_{k}$, такие, что

$$
\int_{D_{j}}(\operatorname{Im} z)^{p \mu-1}|f(z)|^{p} d m_{2}(z) \leqslant \frac{2}{k} \int_{D}(\operatorname{Im} z)^{p \mu-1}|f(z)|^{p} d m_{2}(z),
$$

где $D \subset \Pi$ - квадрат со стороной $\left(x_{1}, x_{k}\right)$, а $D_{j} \subset D$ - квадрат со стороной $J_{j}:=$ $\left(x_{j}, x_{j+1}\right), j=1,2, \ldots, k-1$. Через $\Delta_{j} \subset D_{j}$ обозначим правильный треугольник 
с основанием $J_{j}$. Используя лемму 12 и неравенство $(3.10)$, получим следующее соотношение:

$$
\|f\|_{A_{p, \mu}\left(\Delta_{j}\right)}^{p} \leqslant \frac{c_{1}}{k} \int_{D}(\operatorname{Im} z)^{p \mu-1}|f(z)|^{p} d m_{2}(z) .
$$

Через $p_{j}(z)$ обозначим многочлен Тейлора порядка $l-1$ функции $f^{(-l)}$ с начальной точкой в центре $\Delta_{j}$. Используя теорему 10 с замечанием 2 и соотношение (3.11), находим

$$
\left\|f^{(-l)}-p_{j}\right\|_{L_{q}\left(J_{j}\right)} \leqslant \frac{c_{2}}{k^{1 / p}}\|f\|_{A_{p, \mu}(\Pi)} .
$$

Введем функцию $\psi \in \Pi_{k}^{l}$, полагая $\psi(x)=\operatorname{Im} p_{j}(x)$ при $x \in J_{j}, j=1,2, \ldots$, $k-1$, и $\psi(x)=0$ при $x \in \mathbb{R} \backslash\left[x_{1}, x_{k}\right]$. Функция $\psi$ является искомой. Действительно, из (3.9) и (3.12) получаем

$$
\left\|\operatorname{Im} f^{(-l)}-\psi\right\|_{L_{q}(\mathbb{R})}^{q} \leqslant \frac{c_{3}}{k^{q / p-1}}\|f\|_{A_{p, \mu}(\Pi)}^{q}=\frac{c_{3}}{k^{q(l-\mu)}}\|f\|_{A_{p, \mu}(\Pi)}^{q} .
$$

Лемма доказана.

ДокАЗАТЕЛЬСтво теоремы 3. Согласно лемме 3 нам достаточно получить теорему 3 для $p \in(0,1)$ с $\mu+\frac{1}{p} \notin \mathbb{N}$ и функцией $r \in \mathscr{R}_{n} \cap A_{p, \mu}(\Pi)$ с действительными коэффициентами и полюсами лишь на $\mathbb{R}$. Несложно убедиться, что такие функции $r$ принадлежат $\mathscr{R}_{n}^{l}$ при $l=\left[\mu+\frac{1}{p}\right]$. В силу сделанных ограничений можем определить $q \in(1,+\infty)$, удовлетворяющие условию $\mu+\frac{1}{p}=l+\frac{1}{q}$. Используя лемму 4, находим, что существует действительная функция $\varphi \in \Pi_{m}^{l}$, $m \leqslant n$, удовлетворяющая условию

$$
r^{(-l)}(z)=\frac{1}{\pi} \int_{\mathbb{R}} \frac{\varphi(t) d t}{t-z}, \quad z \in \Pi .
$$

В силу теоремы М. Рисса $r^{(-l)} \in H_{q}(\Pi)$. Очевидно, $\operatorname{Im} r^{(-l)}(z)-$ интеграл Пуассона функции $\varphi$ в П. Следовательно, $\operatorname{Im} r^{(-l)}(x)=\varphi(x)$ для всех $x \in \mathbb{R}$, исключая лишь полюсы $r$ (узлы $\varphi$ ).

Введем наименьшее натуральное $\nu$, для которого $2^{\nu} \geqslant m$. Согласно лемме 6 для каждого $s=0,1,2, \ldots, \nu-1$ найдется действительная функция $\psi_{s} \in \Pi_{2^{s}}^{l}$ такая, что

$$
\left\|\varphi-\psi_{s}\right\|_{L_{q}(\mathbb{R})} \leqslant \frac{c_{1}}{\left(2^{s}\right)^{l-\mu}}\|r\|_{A_{p, \mu}(\Pi)} .
$$

Положим $\varphi_{s}=\psi_{s}-\psi_{s-1}, s=1,2, \ldots, \nu$, где считаем $\psi_{\nu}=\varphi$. Напомним также, что $\varphi_{0} \equiv 0$. Очевидно, $\varphi_{s} \in \Pi_{2^{s+1}}^{l}$ и

$$
\varphi=\varphi_{1}+\varphi_{2}+\cdots+\varphi_{\nu}
$$

всюду на $\mathbb{R}$. Из (3.14) находим также, что

$$
\left\|\varphi_{s}\right\|_{L_{q}(\mathbb{R})} \leqslant \frac{c_{2}}{\left(2^{s}\right)^{l-\mu}}\|r\|_{A_{p, \mu}(\Pi)} .
$$

Сейчас мы можем получить нужную оценку $\|r\|_{L_{\lambda}(\mathbb{R})}$. Действительно, согласно (3.13) и (3.15) получаем

$$
\|r\|_{L_{\lambda}(\mathbb{R})}^{\lambda}=\|r\|_{H_{\lambda}(\Pi)}^{\lambda}=\left\|\left(r^{(-l)}\right)^{(l)}\right\|_{H_{\lambda}(\Pi)}^{\lambda} \leqslant \sum_{s=1}^{\nu}\left\|\frac{l !}{\pi} \int_{\mathbb{R}} \frac{\varphi_{s}(t) d t}{(t-z)^{l+1}}\right\|_{H_{\lambda}(\Pi)}^{\lambda} .
$$


Мы продолжим оценку, применив лемму 5 при $k=l$ и неравенство (3.16). В итоге получим

$$
\begin{aligned}
\|r\|_{L_{\lambda}(\mathbb{R})}^{\lambda} & \leqslant c_{3} \sum_{s=1}^{\nu} \frac{\left(2^{s+1}\right)^{l \lambda}}{\left(2^{s}\right)^{\lambda(l-\mu)}}\|r\|_{A_{p, \mu}(\Pi)}^{\lambda}=c_{4}\|r\|_{A_{p, \mu}(\Pi)}^{\lambda} \sum_{s=1}^{\nu}\left(2^{\lambda \mu}\right)^{s} \\
& \leqslant c_{5}\left(2^{\nu}\right)^{\lambda \mu}\|r\|_{A_{p, \mu}(\Pi)}^{\lambda} \leqslant c_{6} n^{\lambda \mu}\|r\|_{A_{p, \mu}(\Pi)}^{\lambda} .
\end{aligned}
$$

Теорема доказана.

\section{§ 4. Неравенства типа Бернштейна для квазинорм производных рациональных функций и доказательство обратной теоремы}

Некоторые частные случаи следующей теоремы 8 получены в [5]-[7].

ТЕОрема 8. Пусть $p, \mu-$ положительные числа такие, что $\mu+\frac{1}{p} \notin \mathbb{N}$, $s \in \mathbb{N} u \frac{1}{\lambda}=\mu+s+\frac{1}{p}$. Ecлu $r \in \mathscr{R}_{n} \cap A_{p, \mu}(\Pi)$, mo

$$
\left\|r^{(s)}\right\|_{H_{\lambda}} \leqslant c(p, \mu, s) n^{\mu+s}\|r\|_{A_{p, \mu}} .
$$

ДокАЗАТЕЛЬСтво. Поскольку $r \in A_{p, \mu}$, то (см. 2$) r^{(s)} \in A_{p, \mu+s}$ и

$$
\left\|r^{(s)}\right\|_{A_{p, \mu+s}} \leqslant c_{1}(p, \mu, s)\|r\|_{A_{p, \mu}} .
$$

Очевидно также, что $r^{(s)}-$ рациональная функция степени не выше $(s+1) n$. Остается применить к функции $r^{(s)}$ теорему $3 \mathrm{c} \mu+s$ вместо $\mu$ и воспользоваться неравенством (4.1).

Квадраты семейства $\mathscr{F}_{1 / 2}$ (см. $\left.\S 2\right)$ будем нумеровать в каком-либо порядке: $\mathscr{F}_{1 / 2}=\left\{Q_{k}\right\}_{k=1}^{\infty}$. Через $d\left(Q_{k}\right)$ (в соответствии с п. 5.1) обозначаем длину диагонали квадрата $Q_{k}$.

Лемма 7. Пусть $f \in H_{p}(\Pi), 0<p<\infty$. Тогда последовательность

$$
\delta_{k}(f, p):=d\left(Q_{k}\right)^{1 / p}\|f\|_{C\left(Q_{k}\right)}, \quad k=1,2,3, \ldots,
$$

является бесконечно малой. При этом если квадраты $Q_{k}$ семейства $\mathscr{F}_{1 / 2}$ пронумерованы таким образом, что указанная последовательность не возpacmaem, mo

$$
\delta_{k}(f, p) \leqslant \frac{c(p)}{k^{1 / p}}\|f\|_{H_{p}}, \quad k=1,2,3, \ldots
$$

Лемма 7 для круга имеется в [13], [5] и [14]. Ее доказательство для полуплоскости аналогично.

ДОКАЗАТЕЛЬСТВо ТЕОРЕМЫ 4. Положим $s=\max \{1,[\alpha]+1\}, \frac{1}{\lambda}=\mu+s+\frac{1}{p}$ и, кроме того,

$$
\delta_{k}=\delta_{k}\left(r^{(s)}, \lambda\right)=d\left(Q_{k}\right)^{1 / \lambda}\left\|r^{(s)}\right\|_{C\left(Q_{k}\right)}, \quad k=1,2,3, \ldots
$$

Используя неравенство (4.1) и лемму 11, получаем

$$
\sum_{k=1}^{\infty} \delta_{k}^{p} \leqslant c_{1}\|r\|_{A_{p, \mu}}^{p} .
$$


Значит, последовательность $\left\{\delta_{k}\right\}_{k=1}^{\infty}$ является бесконечно малой, и мы можем считать, что квадраты $Q_{k}$ семейства $\mathscr{F}_{1 / 2}$ пронумерованы так, что указанная последовательность не возрастает. В таком случае

$$
\delta_{k} \leqslant \frac{c_{2}}{k^{1 / p}}\|r\|_{A_{p, \mu}}, \quad k=1,2,3, \ldots .
$$

Используя теорему 8 и лемму 7 , находим также, что

$$
\delta_{k}=c_{3} \frac{n^{\mu+s}}{k^{1 / \lambda}}\|r\|_{A_{p, \mu}}, \quad k=1,2,3, \ldots
$$

Из (4.2) и (4.3) получаем

$$
\sum_{k=1}^{\infty} \delta_{k}^{\tau} \leqslant c_{4} n^{\tau(\alpha+\mu)}\|r\|_{A_{p, \mu}}^{\tau} .
$$

Снова применяя лемму 11, убеждаемся, что последнее неравенство равносильно утверждению теоремы 4.

При доказательстве необходимости из теоремы 1 (обратной теоремы) будет применяться метод вещественной интерполяции. Необходимые сведения имеются в [10].

Для $\theta \in(0,1)$ и $0<q \leqslant \infty$ через $(\cdot, \cdot)_{\theta, q}$ обозначаем интерполяционный функтор Я. Петре.

Введем $\mathfrak{R}_{q}^{\beta}, \beta>0, q>0,-$ аппроксимационное пространство функций $f \in$ $A_{p, \mu}(\Pi)$, для которых конечна квазинорма

$$
\|f\|_{\mathfrak{R}_{q}^{\beta}}=\left[\sum_{k=1}^{\infty} \frac{1}{k}\left(k^{\beta} R_{n}(f)_{p, \mu}\right)^{q}\right]^{1 / q} .
$$

В обозначении пространства $\mathfrak{R}_{q}^{\beta}$ мы опускаем $p$ и $\mu$, так как эти параметры до конца данного параграфа считаются фиксированными.

Лемма 8. Пусть $0<\beta_{0}<\beta_{1}<\infty, 0<\theta<1, \beta=(1-\theta) \beta_{0}+\theta \beta_{1} u$ $q, q_{0}, q_{1} \in(0, \infty)$. Тогда

$$
\left(\mathfrak{R}_{q_{0}}^{\beta_{0}}, \mathfrak{R}_{q_{1}}^{\beta_{1}}\right)_{\theta, q}=\mathfrak{R}_{q}^{\beta} .
$$

Эта лемма является частным случаем теоремы 7.1 .8 из [10].

Лемма 9. Пусть $1<\tau_{0}<\tau_{1}<\infty, \alpha_{0}<\frac{1}{\tau_{0}}, \alpha_{1}<\frac{1}{\tau_{1}}, \alpha_{0} \neq \alpha_{1}, 0<\theta<1$ u

$$
\alpha=(1-\theta) \alpha_{0}+\theta \alpha_{1}, \quad \frac{1}{\tau}=\frac{1-\theta}{\tau_{0}}+\frac{\theta}{\tau_{1}} .
$$

Тогда $\left(B_{\tau_{0}}^{\alpha_{0}}, B_{\tau_{1}}^{\alpha_{1}}\right)_{\theta, \tau}=B_{\tau}^{\alpha}$.

Лемма 9 является частным случаем теоремы 6.4 .5 из [10]. Читателей не должно смущать то обстоятельство, что теория пространств Бесова в [10] изложена с иной точки зрения, чем в настоящей статье.

ДокаЗАТЕЛЬСтво теоремы 1. Необходимость. Согласно введенным выше обозначениям нам нужно доказать, что

$$
\mathfrak{R}_{\tau}^{\alpha+\mu} \hookrightarrow B_{\tau}^{\alpha} .
$$


Применяя теорему 4 и метод С.Н. Бернштейна доказательства обратных теорем теории аппроксимации, легко убедиться в справедливости вложения

$$
\mathfrak{R}_{\min \{\tau, 1\}}^{\alpha+\mu} \hookrightarrow B_{\tau}^{\alpha} .
$$

Вложения (4.4) и (4.5) совпадают при $0<\tau \leqslant 1$.

Пусть сейчас $\tau>1$. Выберем $-\mu<\alpha_{0}<\alpha_{1}<\infty$ и $0<\theta<1$, удовлетворяющие условиям $\alpha=(1-\theta) \alpha_{0}+\theta \alpha_{1}, \frac{1}{\tau_{1}}=\alpha_{1}+\mu+\frac{1}{p}<1$. Тогда будем иметь также, что $\frac{1}{\tau_{0}}=\alpha_{0}+\mu+\frac{1}{p}<1$ и

$$
\alpha+\mu=(1-\theta)\left(\alpha_{0}+\mu\right)+\theta\left(\alpha_{1}+\mu\right), \quad \frac{1}{\tau}=\frac{1-\theta}{\tau_{0}}+\frac{\theta}{\tau_{1}} .
$$

Согласно (4.5) выполняются вложения

$$
\mathfrak{R}_{1}^{\alpha_{s}+\mu} \hookrightarrow B_{\tau_{s}}^{\alpha_{s}}, \quad s=0,1 .
$$

Следовательно,

$$
\left(\mathfrak{R}_{1}^{\alpha_{0}+\mu}, \mathfrak{R}_{1}^{\alpha_{1}+\mu}\right)_{\theta, \tau} \hookrightarrow\left(B_{\tau_{0}}^{\alpha_{0}}, B_{\tau_{1}}^{\alpha_{1}}\right)_{\theta, \tau}
$$

Остается воспользоваться леммами 8 и 9. Теорема 1 доказана полностью.

ЗАмечАниЕ 1 . Существенность ограничения $\frac{1}{p}+\mu \notin \mathbb{N}$ в теореме 3 подтверждается на примере семейства рациональных функций степени $2 l$

$$
r_{\varepsilon}(z)=[(z+i)(z+i \varepsilon)]^{-l}, \quad l:=\frac{1}{p}+\mu \in \mathbb{N},
$$

зависящих от параметра $\varepsilon \in\left(0, \frac{1}{2}\right]$. Несложные вычисления показывают (случай $\mu=\frac{1}{p}$ рассмотрен в [7]), что при $\varepsilon \in\left(0, \frac{1}{2}\right]$

$$
\left\|r_{\varepsilon}\right\|_{L_{\lambda}(\mathbb{R})} \asymp\left(\log \frac{1}{\varepsilon}\right)^{1 / p+\mu}, \quad\left\|r_{\varepsilon}\right\|_{A_{p, \mu}(\Pi)} \asymp\left(\log \frac{1}{\varepsilon}\right)^{1 / p} .
$$

Поскольку $\left\|r_{\varepsilon}\right\|_{L_{\lambda}(\mathbb{R})}$ при $\varepsilon \rightarrow+0$ растет существенно быстрее, чем $\left\|r_{\varepsilon}\right\|_{A_{p, \mu}(\Pi)}$, то теорема 3 не выполняется при $\frac{1}{p}+\mu \in \mathbb{N}$.

Аналогично можно показать, что ограничение $\frac{1}{p}+\mu \notin \mathbb{N}$ существенно и в теоремах 4 и 8. Отсюда следует существенность ограничения $\frac{1}{p}+\mu \notin \mathbb{N}$ и для необходимости из теоремы 1.

\section{§ 5. Приложение. Некоторые результаты о пространствах Бергмана и Смирнова}

Цель настоящего параграфа - получить вспомогательные утверждения о пространствах Бергмана и В. И. Смирнова в области. Эти утверждения применялись нами при доказательстве основных результатов этой статьи. Теоремы 9 и 10 (см. ниже) получены в бо́льшей общности, чем требовалось для доказательства теоремы 3, так как они представляют самостоятельный интерес. 
5.1. Пространства Бергмана в области и разбиение типа Уитни. Пусть $G \subset \mathbb{C}$ - область, отличная от $\mathbb{C} ; \partial G$ - граница $G ; \bar{G}=G \cup \partial G$ - замыкание области $G ; \rho(z, \partial G)$ - расстояние от точки $z$ до границы $\partial G$. В $\S 1$ для положительных чисел $p$ и $\mu$ введены пространства Лебега $L_{p, \mu}(\Pi)$ и Бергмана $A_{p, \mu}(\Pi)$. Аналогично вводятся пространства Лебега $L_{p, \mu}=L_{p, \mu}(G)$ и Бергмана $A_{p, \mu}=A_{p, \mu}(G)$ в области $G$. При этом $\operatorname{Im} z$ заменяется на $\rho(z, \partial G)$.

Лемма 10. Если $f \in A_{p, \mu}(G)$, mo

$$
|f(z)| \leqslant c(p, s, \mu) \frac{\|f\|_{A_{p, \mu}}}{(\rho(z, \partial G))^{\mu+1 / p}}, \quad z \in G .
$$

Доказательство основано на субгармоничности функции $|f(z)|^{p}$ (см., например, утверждение 1.1 из [12]).

С использованием леммы 10 доказывается, в частности, полнота пространства $A_{p, \mu}(G)$. Если $G$ - жорданова область со спрямляемой границей, то, как следует из теоремы 1 работы [15], множество алгебраических многочленов всюду плотно в $A_{p, \mu}(G)$.

Пусть $K, \Gamma$ - некоторые множества в $\mathbb{C}$ и $\rho(K, \Gamma)$ - расстояние между $K$ и $\Gamma$, т.е. $\rho(K, \Gamma)=\inf \{|z-\xi|: z \in K, \xi \in \Gamma\}$. Через $d(K)$ и $d_{0}(K)$ обозначим соответственно диаметр наименьшего замкнутого круга, содержащего $K$, и диаметр наибольшего открытого круга, содержащегося в $K$.

Пусть $G$ - область, отличная от $\mathbb{C}$. Семейство $\mathscr{Z}$ односвязных замкнутых областей $Q$ с кусочно гладкой границей называется разбиением типа Уитни области $G$, если выполняются следующие условия:

(i) любые две области семейства $\mathscr{Z}$ могут пересекаться лишь по границам;

(ii) объединение всех областей семейства $\mathscr{Z}$ есть $G$;

(iii) существуют постоянные $c_{1}$ и $c_{2}$ такие, что для любой области $Q \subset \mathscr{Z}$ выполняются неравенства

$$
c_{1} \rho(Q, \partial G) \leqslant d_{0}(Q) \leqslant d(Q) \leqslant c_{2} \rho(Q, \partial G) .
$$

Пример такого разбиения области $G$ дают квадраты Уитни (см. [16; гл. VI]) с $c_{1}=\frac{\sqrt{2}}{8}$ и $c_{2}=1$. Если $G=\Pi$, то построенное в $\S 2$ семейство $\mathscr{F}_{\theta}, 0<\theta<1$, является разбиением типа Уитни с $c_{1}=\frac{1}{\theta}-1, c_{2}=\sqrt{2}\left(\frac{1}{\theta}-1\right)$.

Если $K$ - компакт в $\mathbb{C}$, то $C(K)$ обозначает множество комплексных непрерывных функций на $K$. Для $f \in C(K)$ полагаем $\|f\|_{C(K)}=\max _{z \in K}|f(z)|$.

Лемма 11. Пусть р и

$$
c_{3}\|f\|_{A_{p, \mu}}^{p} \leqslant \sum_{Q \in \mathscr{Z}}\left(d(Q)^{\mu+1 / p}\|f\|_{C(Q)}\right)^{p} \leqslant c_{4}\|f\|_{A_{p, \mu}}^{p},
$$

где $c_{3}$ и $c_{4}$ зависят от $p, \mu$ и постоянных $c_{1}, c_{2}$ из (5.1).

Доказательство основано (как и в случае леммы 10) на субгармоничности функции $|f(z)|^{p}$. Для круга лемма 11 получена в [17].

Лемма 12. Пусть $p$, а и $\mu$ - положительные числа, D - открытый квадpaт с вершинами в точках $0, a, i a, a+i a u \Delta \subset D$ - правильный треугольник 
с основанием $(0, a)$. Если функиия $f$ аналитична в $D$ u $(\operatorname{Im} z)^{p \mu-1}|f(z)|^{p}$ суммируема в $D$, то

$$
\int_{\Delta} \rho(z, \partial \Delta)^{p \mu-1}|f(z)|^{p} d m_{2}(z) \leqslant c(p, \mu) \int_{D}(\operatorname{Im} z)^{p \mu-1}|f(z)|^{p} d m_{2}(z) .
$$

ДокАЗАТЕЛЬСтво. Поскольку $\rho(z, \partial \Delta) \leqslant \operatorname{Im} z$ при $z \in \Delta$, то для $p \mu \geqslant 1$ неравенство (5.2) выполняется с $c(p, \mu)=1$. Поэтому далее считаем, что $p \mu<1$. Ради удобства будем рассматривать случай $a=2$, так как общий случай сводится к указанному с помощью замены переменной интегрирования в (5.2).

Пусть $z_{0}$ - центр треугольника $\Delta ; \Delta_{0}$ - треугольник с вершинами в точках $z_{0}, 0$ и $2 ; \Lambda=\Delta \backslash \Delta_{0} ; \Lambda_{+}$и $\Lambda_{-}-$соответственно правая и левая половины $\Lambda$, получаемые рассечением $\Lambda$ прямой $x=1$.

Очевидно, $\rho(z, \partial \Delta)=\operatorname{Im} z$ при $z \in \Delta_{0}$ и, следовательно, для доказательства (5.2) нам достаточно убедиться в справедливости неравенства

$$
\int_{\Lambda_{ \pm}} \rho(z, \partial \Delta)^{p \mu-1}|f(z)|^{p} d m_{2}(z) \leqslant c_{1}(p, \mu) \int_{D}(\operatorname{Im} z)^{p \mu-1}|f(z)|^{p} d m_{2}(z) .
$$

Докажем, например, (5.3) для $\Lambda_{-}$. Треугольник $\Lambda_{-} \backslash\{0\}$ представим в виде объединения замкнутых трапеций $T_{k}$, основания которых лежат на прямых $x=\frac{1}{2^{k-1}}$ и $x=\frac{1}{2^{k}}, k=1,2,3, \ldots$, а боковые стороны - на наклонных сторонах треугольника $\Lambda_{-}$. Используя тот же метод, что и при доказательстве леммы 11 , несложно убедиться в справедливости неравенства

$$
\sum_{k=1}^{\infty}\left(d\left(T_{k}\right)\right)^{p \mu+1}\|f\|_{C\left(T_{k}\right)}^{p} \leqslant c_{2}(p, \mu) \int_{D}(\operatorname{Im} z)^{p \mu-1}|f(z)|^{p} d m_{2}(z) .
$$

Из (5.4) немедленно следует (5.3) для $\Lambda_{-}$. Этим лемма доказана.

5.2. Квазиконформное отражение. Здесь будем считать, что $G$ - односвязная ограниченная область, граница которой $\partial G$ является кривой М. А. Лаврентьева. А именно, $\partial G$ - спрямляемая кривая Жордана и для любых точек $\xi_{1}, \xi_{2} \in \partial G$ выполняется неравенство

$$
\left|\Gamma\left(\xi_{1}, \xi_{2}\right)\right| \leqslant \varkappa\left|\xi_{1}-\xi_{2}\right|,
$$

где $\varkappa>1$ - некоторая постоянная, а $\left|\Gamma\left(\xi_{1}, \xi_{2}\right)\right|$ - длина наименьшей $\Gamma\left(\xi_{1}, \xi_{2}\right)$ из двух дуг $\partial G$ с концами $\xi_{1}$ и $\xi_{2}$.

Приведем необходимые нам сведения из теории квазиконформных отображений (см. [18], [19]). Будем считать, что $0 \in G$. Через * будем обозначать квазиконформную инволюцию $\overline{\mathbb{C}}$, удовлетворяющую условиям: $\xi^{* *}=\xi$ при всех $\xi \in \overline{\mathbb{C}}, 0^{*}=\infty, \xi^{*}=\xi$ при $\xi=\partial G$. Отображение ${ }^{*}$ не единственно и его можно выбрать так, чтобы выполнялись условия:

(i) для каждой окрестности $U \subset G$ точки 0 отображение $\xi \mapsto \xi^{*}$ квазиконформно из $\mathbb{C} \backslash\left(U \cup U^{*}\right)$ в себя;

(ii) отображение $\xi \mapsto \xi^{*}$ непрерывно дифференцируемо в $\mathbb{C} \backslash(0 \cup \partial G)$ и для всех $\xi \in \mathbb{C} \backslash\left(U \cup U^{*} \cup \partial G\right)$ выполняются соотношения

$$
\left|\frac{\partial \xi^{*}}{\partial \xi}\right| \leqslant c_{1}, \quad c_{2} \leqslant\left|\frac{\partial \xi^{*}}{\partial \bar{\xi}}\right| \leqslant \frac{1}{c_{2}}
$$

где $c_{1}$ и $c_{2}$ зависят лишь от $\varkappa$ из (5.5) и окрестности $U \subset G$ точки 0. 
Отметим, что для любого множества $D \subset \overline{\mathbb{C}}$ мы полагаем $D^{*}=\left\{\xi^{*}: \xi \in D\right\}$. В дальнейшем будем считать, что отображение * выбрано так, что условия (i), (ii) выполняются. Например, если $G-$ круг $|\xi|<1$, то $\xi^{*}=\frac{1}{\bar{\xi}}$.

Мы будем использовать разбиение $\mathscr{Z}=\left\{Q_{k}\right\}_{k=0}^{\infty}$ области $G$ на области типа Уитни $Q_{k}$, для которого $Q_{0}=\left\{\xi \in G:|\xi| \leqslant \frac{1}{2} \rho(0, \partial G)\right\}$. Такое разбиение легко построить, используя квадраты Уитни.

Из перечисленных свойств отображения * и соотношений (5.1) следует, что последовательности $\rho\left(Q_{k}, \partial G\right), \rho\left(Q_{k}^{*}, \partial G\right), d\left(Q_{k}\right), d\left(Q_{k}^{*}\right), d_{0}\left(Q_{k}\right), d_{0}\left(Q_{k}^{*}\right)$ при $k=1,2,3, \ldots$ являются бесконечно малыми одного порядка малости.

5.3. Интегральное представление. Здесь предполагается, что область $G$ и отображение * такие, как указано в п. 5.2. Рассмотрим замкнутые кривые $\Gamma_{s}=\left\{\xi^{*}:|\xi|=\frac{1}{2^{2-s}} \rho(0, \partial G)\right\}, s=0,1$. Через $\Omega_{0}, \Omega_{1}$ обозначим соответственно замкнутые двусвязные области, заключенные между кривыми $\Gamma_{0}$ и $\Gamma_{1}, \Gamma_{1}$ и $\partial G$. Положим $\Omega=\Omega_{0} \cup \Omega_{1}$. Введем какую-либо гладкую функцию $\eta: \Omega \mapsto[0,1]$, удовлетворяющую условиям: $\eta(\xi)=1$ при $\xi \in \Omega_{1}, \eta(\xi)=0$ при $\xi \in \Gamma_{0}$. Существование такой функции легко обосновать, используя усреднения в смысле В.А. Стеклова. Тогда образ $\Omega$ под действием отображения $\theta(\xi):=\xi^{*} \cdot \eta(\xi)$ совпадает с $\bar{G}$, причем $\theta(\xi)=\xi^{*}$ при $\xi \in \Omega_{1}$.

Теорема 9. Пусть функиия $f$ аналитична в $G, f^{(s)} \in A_{1, s}(G), s \in \mathbb{N}, u$

$$
T_{s-1}(z)=T_{s-1}(z, f)=\sum_{k=0}^{s-1} \frac{f^{(k)}(0)}{k !} z^{k} .
$$

Тогда для $z \in G$ имеет место равенство

$$
f(z)=T_{s-1}(z)-\frac{1}{\pi(s-1) !} \int_{\Omega} f^{(s)}(\theta(\xi))(\xi-\theta(\xi))^{s-1} \frac{\partial \theta(\xi)}{\partial \bar{\xi}} \frac{d m_{2}(\xi)}{\xi-z} .
$$

Эта теорема доказывается точно так же, как лемма 2.3 из [20]. Отличие заключается лишь в том, что вместо замкнутости всех алгебраических многочленов в пространстве В. И. Смирнова $E_{1 /(s+1)}(G)$ следует использовать их же замкнутость в $A_{1, s}(G)$.

Следующая лемма хорошо известна (см., например, [21] или [20]).

Лемма 13. Пусть область $G$ удовлетворяет указанным выше условиям и $\beta>0$. Тогда для любого $\xi \in \mathbb{C} \backslash \partial G$ выполняется неравенство

$$
\int_{\partial G} \frac{|d z|}{|\xi-z|^{\beta+1}} \leqslant \frac{c}{\rho(\xi, \partial G)^{\beta}},
$$

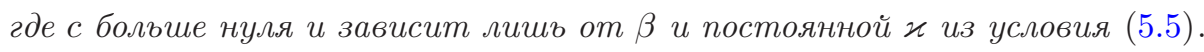

5.4. Пространство В. И. Смирнова. Для спрямляемой кривой $Г$ в $\mathbb{C}$ и $0<p<\infty$ через $L_{p}(\Gamma)$ обозначаем пространство Лебега комплексных измеримых функций $f$ на $\Gamma$ с конечной квазинормой

$$
\|f\|_{L_{p}}=\|f\|_{L_{p}(\Gamma)}=\left(\int_{\Gamma}|f(z)|^{p}|d z|\right)^{1 / p} .
$$

Пусть $G=G_{+}-$односвязная ограниченная область, граница которой $\partial G$ является спрямляемой кривой Жордана; $G_{-}:=\overline{\mathbb{C}} \backslash \bar{G}$. Для $0<p<\infty$ через 
$E_{p}^{ \pm}=E_{p}\left(G_{ \pm}\right)$обозначим пространство В.И. Смирнова функций, аналитических в $G_{ \pm}$(определение и свойства $E_{p}^{ \pm}$см. в [22] и [23]). Если $G-$ круг $|z|<1$, то $E_{p}^{ \pm}$совпадает с пространством Харди $H_{p}^{ \pm}$. Многие свойства пространства $H_{p}^{ \pm}$справедливы и для $E_{p}^{ \pm}$. В частности, если $f \in E_{p}^{ \pm}$, то для почти всех $\xi \in \partial G$ существует $\lim f(z)=: f(\xi)$, когда $z$ принадлежит $G_{ \pm}$и стремится к $\xi$ по некасательным по отношению к $\partial G$ путям. В соответствии с этим функции из $E_{p}^{ \pm}$будем считать определенными как в $G_{ \pm}$, так и почти всюду на $\partial G$. При этом квазинорма $f \in E_{p}^{ \pm}$определяется так: $\|f\|_{E_{p}^{ \pm}}=\|f\|_{L_{p}(\partial G)}$. Отметим, что $f(\infty)=0$ для $f \in E_{p}^{-}$.

Следующая лемма 14 является следствием теоремы Г. Давида (см., например, [21]) о сингулярном интеграле типа Коши.

Лемма 14. Пусть граница $\partial G$ области $G$ является кривой Лаврентьева $u g \in L_{u}(\partial G), 1<u<\infty$. Тогда существуют и единственны функиии $g_{ \pm} \in E_{u}\left(G_{ \pm}\right)$такие, что $g=g_{+}+g_{-}$почти всюду на $\partial G u\left\|g_{ \pm}\right\|_{E_{u}^{ \pm}} \leqslant c\|g\|_{L_{u}}$,

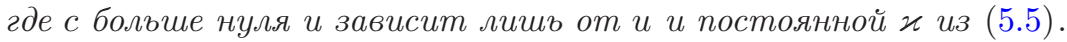

В лемме 15 используется конструкция, описанная в п. 5.2. Эта лемма следует из леммы 2.1 работы [20] и свойств областей $Q_{k}$ и $Q_{k}^{*}, k=0,1,2, \ldots$.

Лемма 15. Пусть $h \in E_{u}\left(G_{-}\right), 0<u<\infty, u$

$$
\delta_{0}=\|h\|_{C\left(Q_{0}^{*}\right)}, \quad \delta_{k}=\left(d\left(Q_{k}^{*}\right)\right)^{1 / u}\|h\|_{C\left(Q_{k}^{*}\right)}, \quad k=1,2,3, \ldots
$$

Тогда:

(i) $\delta_{0} \leqslant c_{1}(G, u)\|h\|_{E_{u}^{-}}$;

(ii) последовательность $\left\{\delta_{k}\right\}_{k=1}^{\infty}$ является бесконечно малой, и если области $Q_{k}, k=1,2,3, \ldots$, перенумерованъ так, что эта последовательность невозрастающая, то

$$
\delta_{k} \leqslant \frac{c_{2}(G, u)}{k^{1 / u}}\|g\|_{E_{u}^{-}}, \quad k=1,2,3, \ldots
$$

5.5. Теорема вложения. Сейчас все подготовлено для доказательства теоремы 10, которая применялась для доказательства теоремы 3 - одного из основных результатов статьи.

Теорема 10. Пусть $p, q u \mu$-положительные числа такие, что

$$
0<p<q<\infty, \quad \mu+\frac{1}{p}-\frac{1}{q}=: l \in \mathbb{N}
$$

u $G$ - односвязная ограниченная область, граница которой является кривой Лаврентьева. Если функиия $f$ аналитична в $G$ и $f^{(l)} \in A_{p, \mu}(G)$, mo $f \in E_{q}(G)$. Кроме того, если $f\left(z_{0}\right)=f^{\prime}\left(z_{0}\right)=\cdots=f^{(l-1)}\left(z_{0}\right)=0$ в некоторой точке $z_{0} \in G, m o$

$$
\|f\|_{E_{q}} \leqslant c\left\|f^{(l)}\right\|_{A_{p, \mu}}
$$

где с больше нуля и не зависит от $f$.

ЗАмечАниЕ 2. Анализ приведенного далее доказательства теоремы 10 показывает, что если $z_{0}$ - центр наибольшего круга, вписанного в $\bar{G}$ (или один из таких центров, если их несколько), то можно считать, что постоянная в (5.6) зависит лишь от $p, q, \mu$ и постоянной $\varkappa$ из (5.5). 
ДоКАЗАТЕЛЬСТВо теОРЕМы 10. Без ограничения общности будем считать, что $0 \in G$ и $z_{0}=0$. В силу замкнутости множества всех алгебраических многочленов в $E_{q}$ и $A_{p, \mu}$ нам достаточно получить неравенство (5.6) для любого алгебраического многочлена $f$, удовлетворяющего условиям $f(0)=f^{\prime}(0)=$ $\cdots=f^{(l-1)}(0)=0$. При таком ограничении на функцию $f$ включения $f \in E_{q}$ и $f^{(l)} \in A_{p, \mu}$ очевидны. Мы будем применять теорему 9 и построенные в п. 5.2 множества $Q_{k}, k=0,1,2, \ldots$.

Рассмотрим сначала случай $1<q<\infty$. Через $q^{\prime}$ обозначим показатель, сопряженный $q$, т.е. $\frac{1}{q^{\prime}}+\frac{1}{q}=1$. В силу двойственности $\left(L_{q}(\partial G)\right)^{\prime}=L_{q^{\prime}}(\partial G)$ существует функция $g \in L_{q^{\prime}}(\partial G)$ такая, что $\|g\|_{L_{q^{\prime}}}=1$ и

$$
\|f\|_{E_{q}(G)}=\|f\|_{L_{q}(\partial G)}=\int_{\partial G} f(z) g(z) d z .
$$

Согласно лемме 14 существуют функции $g_{ \pm} \in E_{q}\left(G_{ \pm}\right)$, удовлетворяющие условиям: $\left\|g_{ \pm}\right\|_{E_{q}^{ \pm}} \leqslant c_{1}$ и $g=g_{+}+g_{-}$почти всюду на $\partial G$. Поскольку $f g_{+} \in E_{1}\left(G_{+}\right)$, то в силу обобщенной теоремы Коши (см. [22], [23]) $\int_{\partial G} f(z) g_{+}(z) d z=0$. По-
этому с учетом (5.7) получим

$$
\|f\|_{E_{q}}=\int_{\partial G} f(z) g_{-}(z) d z .
$$

Используя последнее равенство, теорему 9 при $s=l$, теорему Фубини и интегральную формулу Коши, получим

$$
\|f\|_{E_{q}}=\frac{2 i}{l !} \int_{\Omega} f^{(l)}(\theta(\xi)) g_{-}(\xi)(\xi-\theta(\xi))^{l-1} \frac{\partial \theta(\xi)}{\partial \bar{\xi}} d m_{2}(\xi) .
$$

Далее мы применим лемму 15 при $h=g_{-}$и $u=q^{\prime}$. Будем считать, что области $\left\{Q_{k}\right\}_{k=1}^{\infty}$ пронумерованы таким образом, что соответствующая последовательность $\left\{\delta_{k}\right\}_{k=1}^{\infty}$ невозрастающая. Учитывая, что равномерно по $\xi \in Q_{k}$ и $k=1,2,3, \ldots$ выполняются соотношения $|\xi-\theta(\xi)| \asymp d\left(Q_{k}\right) \asymp d\left(Q_{k}^{*}\right)$, из (5.8) получим

$$
\|f\|_{E_{q}} \leqslant c_{1}\left(d\left(Q_{0}\right)\right)^{l+1 / q}\left\|f^{(l)}\right\|_{C\left(Q_{0}\right)}+c_{2} \sum_{k=1}^{\infty}\left(d\left(Q_{k}\right)\right)^{l+1 / q}\left\|f^{(l)}\right\|_{C\left(Q_{k}\right)} \cdot k^{-1 / q^{\prime}} .
$$

Заметим, что согласно условию $l+\frac{1}{q}=\mu+\frac{1}{p}$ и согласно лемме 10 первое слагаемое из правой части последнего неравенства не превышает $c_{3}\left\|f^{(l)}\right\|_{A_{p, \mu}}$. Поэтому для завершения рассматриваемого случая $1<q<\infty$ нам достаточно убедиться в справедливости неравенства

$$
\sum_{k=1}^{\infty}\left(d\left(Q_{k}\right)\right)^{\mu+1 / p}\left\|f^{(l)}\right\|_{C\left(Q_{k}\right)} \cdot k^{-1 / q^{\prime}} \leqslant c_{4}\left\|f^{(l)}\right\|_{A_{p, \mu}} .
$$

В случае $0<p \leqslant 1<q<\infty$ это неравенство следует из лемм 2 и 11 . Если же $1<p<q<\infty$, то к левой части (5.9) необходимо применить неравенство Гёльдера с показателями $p$ и $p^{\prime}, \frac{1}{p^{\prime}}+\frac{1}{p}=1$, и снова воспользоваться леммой 11 .

Рассмотрим сейчас случай $0<q \leqslant 1$. Положим $\nu=\left[\frac{1}{q}\right]+1$. Тогда для $z \in \bar{G}$ справедливо равенство

$$
f(z)=-\frac{\nu !}{\pi(l+\nu-1) !} \int_{\Omega} f^{(l)}(\theta(\xi))(\xi-\theta(\xi))^{l+\nu-1} \frac{\partial \theta(\xi)}{\partial \bar{\xi}} \frac{d m_{2}(\xi)}{(\xi-z)^{\nu+1}}
$$


Для получения (5.10) при $z \in G$ нужно применить теорему 9 к $f^{(-\nu)}$ (т.е. к $\nu$-й первообразной функции $f)$ и $s=l+\nu$, затем полученное интегральное представление для $f^{(-\nu)}$ продифференцировать $\nu$ раз. Тогда справедливость равенства (5.6) для $z \in \bar{G}$ следует из непрерывности $f$ в $\bar{G}$ и равномерной ограниченности по $z \in G$ и $\xi \in \Omega$ подынтегральной функции из правой части (5.10).

Выберем какие-либо точки $\xi_{0} \in \Omega_{0}$ и $\xi_{k} \in Q_{k}^{*}, k=1,2, \ldots$. Тогда из $(5.10)$ получим, что при $z \in \bar{G}$ выполняется неравенство

$$
|f(z)| \leqslant c_{5} \sum_{k=0}^{\infty}\left(d\left(Q_{k}\right)\right)^{l+\nu+1}\left\|f^{(l)}\right\|_{C\left(Q_{k}\right)} \cdot \frac{1}{\left|\xi_{k}-z\right|^{\nu+1}} .
$$

Согласно построению множеств $Q_{k}$ и выбору точек $\xi_{k}$ имеем $d\left(Q_{k}\right) \asymp \rho\left(\xi_{k}, \partial G\right)$ при $k=1,2,3, \ldots$. Поэтому, учитывая, что $0<q \leqslant 1, q(\nu+1)>1$, и используя (5.11) и лемму 13, получим

$$
\int_{\partial G}|f(z)|^{q}|d z| \leqslant c_{6} \sum_{k=0}^{\infty}\left(\left(d\left(Q_{k}\right)\right)^{l+1 / q}\left\|f^{(l)}\right\|_{C\left(Q_{k}\right)}\right)^{q} .
$$

Поскольку $0<p<q$, то, используя леммы 2 и 11, из (5.12) получаем (5.6). Теорема 10 полностью доказана.

\section{Список литературы}

[1] R. Rochberg, "Decomposition theorems for Bergman spaces and their applications", Operators and function theory (Lancaster, 1984), NATO Adv. Sci. Inst. Ser. C Math. Phys. Sci., 153, Reidel, Dordrecht, 1985, 225-277.

[2] K. Zhu, Spaces of holomorphic functions in the unit ball, Grad. Texts in Math., 226, Springer-Verlag, New York, 2005.

[3] А. А. Пекарский, "Классы аналитических функций, определяемые наилучшими рациональными приближениями в $H_{p} "$, Матем. сб., 127(169):1(5) (1985), 3-20; англ. пер.: А. A. Pekarskiı̌, "Classes of analytic functions determined by best rational approximations in $H_{p}$ ", Math. USSR-Sb., 55:1 (1986), 1-18.

[4] R. R. Coifman, R. Rochberg, "Representation theorems for holomorphic and harmonic functions in $L^{p}$ ", Representation theorems for Hardy spaces, Astérisque, 77, Soc. Math. France, Paris, 1989, 11-66.

[5] E. Dyn'kin, "Rational functions in Bergman spaces", Complex analysis, operators, and related topics, Oper. Theory Adv. Appl., 113, Birkhüser, Basel, 2000, 77-94.

[6] В.Р. Мисюк, "Неравенства типа Бернштейна для производных рациональных функций относительно плоской меры”, Тр. ИМ НАН Беларуси, 9 (2001), 105-108.

[7] Т. С. Мардвилко, "Неравенство для квазинорм рациональной функции относительно линейной и плоской мер и его приложения", Вестн. НАН Беларуси. Сер. физ.-матем. наук, 1 (2010), 41-48.

[8] А. А. Пекарский, Г. Шталь, "Неравенства типа Бернштейна для производных рациональных функций в пространствах $L_{p}$ при $p<1$ ”, Матем. сб., 186:1 (1995), 119-130; англ. пер.: А. А. Pekarskii, H. Stahl, "Bernstein type inequalities for derivatives of rational functions in $L_{p}$ spaces for $p<1$ ", Sb. Math., 186:1 (1995), 121-131.

[9] Т. С. Мардвилко, А.А. Пекарский, "Рациональная аппроксимация в пространстве Бергмана в полуплоскости", Современные проблемы теории функиий и их приложения, Материалы 15-й Саратовской зимней школы, Изд-во Саратовского ун-та, Саратов, 2010, 113-115. 
[10] Й. Берг, Й. Лефстрем, Интерполяиионные пространства. Введение, Мир, М., 1980; пер. с англ.: J. Bergh, J. Löfström, Interpolation spaces. An introduction, Springer-Verlag, Berlin-Heidelberg-New York, 1976.

[11] Дж. Гарнетт, Ограниченные аналитические функиии, Мир, М., 1984; пер. с англ.: J.B. Garnett, Bounded analytic functions, Pure Appl. Math., 96, Academic Press, New York-London, 1981.

[12] H. Hedenmalm, B. Korenblum, K. Zhu, Theory of Bergman spaces, Grad. Texts in Math., 199, Springer-Verlag, New York, 2000.

[13] А. А. Пекарский, "Обобщенная рациональная аппроксимация в круге", Изв. АН БССР. Сер. физ.-матем. наук, 6 (1990), 9-14.

[14] A. A. Pekarskii, "Approximation by rational functions with free poles", East J. Approx., 13:3 (2007), 227-319; "Corrigendum", East J. Approx., 13:4 (2007), 483.

[15] J. E. Brennan, "Point evaluations, invariant subspaces and approximation in the mean by polynomials", J. Funct. Anal., 34:3 (1979), 407-420.

[16] И. М. Стейн, Сингулярные интегралы и дифференциальные свойства функиий, Мир, М., 1973; пер. с англ.: Е. М. Stein, Singular integrals and differentiability properties of functions, Princeton Math. Ser., 30, Princeton Univ. Press, Princeton, NJ, 1970.

[17] Ф.А. Шамоян, "Теоремы вложения и характеристика следов в пространствах $H^{p}\left(U^{n}\right), 0<p<\infty "$, Матем. сб., 107(149):3(11) (1978), 446-462; англ. пер.: F. A. Samojan, "Embedding theorems and a characterization of traces in the spaces $H^{p}\left(U^{n}\right), 0<p<\infty "$, Math. USSR-Sb., 35:5 (1979), 709-725.

[18] Л. В. Альфорс, Лекции по квазиконформным отображениям, Мир, М., 1969; пер. с англ.: L. V. Ahlfors, Lectures on quasiconformal mappings, Van Nostrand, Toronto, ON-New York-London, 1966.

[19] В. И. Белый, "Современные методы геометрической теории функции комплексного переменного в задачах аппроксимации”, Алгебра и анализ, 9:3 (1997), 3-40; англ. пер.: V.I. Belyг̌, "Modern methods of the geometric theory of functions of a complex variable in approximation problems", St. Petersburg Math. J., 9:3 (1998), 421-453.

[20] А.А. Пекарский, "Рациональные приближения функций с производными из пространства В.И. Смирнова", Алгебра и анализ, 13:2 (2001), 165-190; англ. пер.: A.A. Pekarskii, "Rational approximation of functions with derivatives in a V.I. Smirnov space", St. Petersburg Math. J., 13:2 (2002), 281-300.

[21] G. David, "Opérateurs intégraux singuliers sur certaines courbes du plan complexe", Ann. Sci. École Norm. Sup. (4), 17:1 (1984), 157-189.

[22] И. И. Привалов, Граничные свойства аналитических функиий, ГИТТЛ, М.-Л., 1950.

[23] P. L. Duren, Theory of $H^{p}$ spaces, Academic Press, New York-London, 1970.

T. С. Мардвилко (T.S. Mardvilko)

Белорусский государственный университет, г. Минск

Поступила в редакцию

E-mail: mardvilko@mail.ru

17.05.2010

\section{А. А. Пекарский (А. A. Pekarskii)}

Белорусский государственный университет, г. Минск

E-mail: pekarskii@gmail.com 